\title{
Determinant of the SU(N) caloron with nontrivial holonomy
}

\author{
Sergey Slizovskiy ${ }^{1 a, b}$ \\ a St.Petersburg INP, Gatchina, 188 300, St.Petersburg, Russia \\ ${ }^{b}$ Department of Theoretical Physics, Uppsala University, P.O. Box 803, S-75108, Uppsala, Sweden
}

\begin{abstract}
The 1-loop quantum weight of the SU(N) KvBLL caloron with nontrivial holonomy is calculated. The latter is the most general self-dual solution with unit topological charge in the $4 \mathrm{~d}$ Yang-Mills theory with one compactified dimension (finite temperature).
\end{abstract}

\section{Introduction}

The finite temperature field theory is defined by considering the Euclidean space-time which is compactified in the 'time' direction whose inverse circumference is a temperature $T$, with the usual periodic boundary conditions for boson fields and antiperiodic conditions for fermion fields. In particular, the gauge field is periodic in time, so the theory is no longer invariant under arbitrary gauge transformations. Only timeperiodic gauge transformations are allowed and hence the number of gauge invariants increases. The new invariant is the holonomy or the eigenvalues of the Polyakov line that winds along the compact 'time' direction [1]:

$$
L=\left.\mathrm{P} \exp \left(\int_{0}^{1 / T} d t A_{4}\right)\right|_{|\vec{x}| \rightarrow \infty} .
$$

This invariant together with the topological charge and the magnetic charge can be used for the classification of the field configurations [2] , its zero vacuum average is one of the common criteria of confinement.

A generalization of the usual Belavin-Polyakov-Schwartz-Tyupkin (BPST) instantons [3] for arbitrary temperatures and holonomies is the Kraan-van Baal-Lee-Lu (KvBLL) caloron with non-trivial holonomy $[4,5,6]$. It is a self-dual electrically neutral configuration with unit topological charge and arbitrary holonomy. This solution was constructed by Kraan and van Baal [4] and Lee and Lu [6] for the SU(2) gauge group and in [5] for the general $S U(N)$ case; it has been named the KvBLL caloron (recently the exact solutions of higher topological charge were constructed and discussed [7, 8]).

\footnotetext{
${ }^{1}$ Sergey.Slizovskiy@teorfys.uu.se
} 

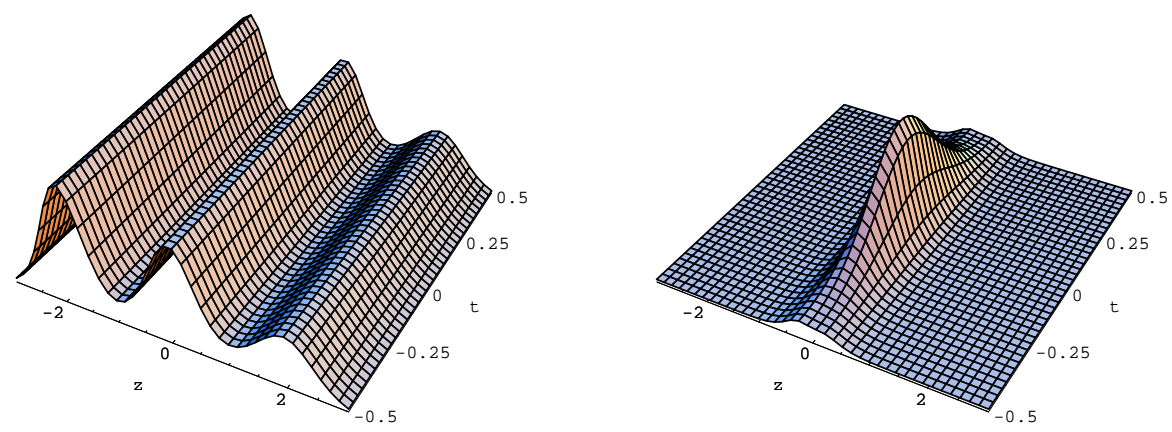

Figure 1: The action density of the $S U(3)$ KvBLL caloron as function of $z, t$ at fixed $x=y=0$, eigenvalues of $A_{4}$ at spatial infinity are $\mu_{1}=-0.307 T, \mu_{2}=-0.013 T, \mu_{3}=$ $0.32 \mathrm{~T}$. It is periodic in $t$ direction. At large dyon separation the density becomes static (left, $\varrho_{1,2}=1 / T, \varrho_{3}=2 / T$ ). As the separation decreases the action density becomes more like a $4 d$ lump (right, $\varrho_{1,2}=1 /(3 T), \varrho_{3}=2 /(3 T)$ ). The axes are in units of inverse temperature $1 / T$.

There is a plenty of lattice studies supporting the presence of these solution [9], see also [10] for a very brief review. In a recent paper [11] the caloron ensemble was studied analytically, although some contributions were neglected there, the results are in very good agreement with phenomenology.

The holonomy is called 'trivial' if the Polyakov loop (1) acquires values belonging to the group center $Z(N)$. For this case the KvBLL caloron reduces to the periodic Harrington-Shepard [12] caloron known before. The latter is purely an SU(2) configuration and its quantum weight was studied in detail by Gross, Pisarski and Yaffe [2].

The KvBLL caloron in the theory with $S U(N)$ gauge group on the space $R^{3} \times S^{1}$ can be interpreted as a composite of $N$ distinct fundamental monopoles (dyons) [13][14] (see fig. 1 ). As was proven in [5, 17], the exact KvBLL gauge field reduces to a superposition of BPS dyons, when the separation $\varrho_{i}$ between dyons is large (in units of inverse temperature). On the contrary, the KvBLL caloron reduces to the usual BPST instanton, when the distances $\varrho_{l}$ between all the dyons become small compared to the inverse non-triviality of holonomy.

We refer the reader to the papers $[5,17]$ for the detailed discussion and construction of the caloron solutions, to the original works [4] for the $S U(2)$ case and to further works on higher topological charge solutions $[7,8]$.

This paper is in the series of papers $[15,16,17,18,19]$ where we calculate the functional determinant for KvBLL calorons with nontrivial holonomy [4,6] in the finitetemperature Yang-Mills theory.

Here we calculate the 1-loop gluonic and ghost functional determinants for the case of an arbitrary $S U(N)$ gauge group. The calculation is performed in the limit of far separated dyon constituents and up to an overall numerical constant. The constant for the gluonic determinant remains known only for the SU(2) case [15] ${ }^{2}$.

We find new 3-particle interactions arising between constituent dyons due to the glu-

\footnotetext{
${ }^{2}$ In the previous paper [17] we have proved that the corresponding constant is zero for the fundamental-representation determinant, but for the adjoint representation the constant is not expected to vanish.
} 
onic determinant. These terms were not present in the fermionic (fundamental representation) determinant and also vanished in the $S U(2)$ gluonic determianant.

Similar to our previous results, the determinant is infrared divergent, the leading divergence is proportional to the volume or the system, and there are of course several subleading divergent terms. It is not surprising and was known long ago $[25,2]$ that non-trivial holonomy increases the effective action by a factor proportional to the volume. Nevertheless that does not make the studies of non-trivial holonomy unphysical, since in the ensemble of many calorons the moduli space integrals can compensate the above divergences.

Since there are subleading divergences coming from the Coulomb tail of dyon fields, it is natural, that the result would also depend on the position of the large ball, with which we make the infra-red cutoff. We will display this dependence, but we note that it is unphysical unless the box in not a real border of plasma region. One could also expect that the quantum corrections will dump the Coulomb tails of dyons.

We present the relevant notations in section 2 and illustrate the notations by the old results. The method of computation is described in 3 and the actual computations are carried out in subsequent sections and appendices. The final result is presented in section 6 .

We do not draw here any physical conclusions on the behaviour of the whole caloron ensemble since that is now a separate business [26], [11]. Our results could be useful for extending the works [26] to the $S U(N>2)$ case and including the corrections due to the non-zero modes to the work [11].

\section{Notations and Review}

Consider the $S U(N)$ YM theory and a caloron solution with the asymptotics ${ }^{3}$

$$
A_{\mu} \stackrel{\vec{x} \rightarrow \infty}{\longrightarrow} 2 \pi \delta_{\mu 4} \operatorname{diag}\left(\mu_{1}, \ldots, \mu_{N}\right) .
$$

For the $S U(2)$ case the standard choice is $\mu_{1}=-\omega ; \mu_{2}=\omega$ where $0 \leq \omega<\frac{1}{2}$. As usual, we set the temperature $T=1$ throughout the computation, and restore the temperature dependence only in the final result.

The caloron can be viewed as composed of dyons (BPS monopoles with $A_{4}$ playing the role of a Higgs field), the inverse dyon size $v$ being defined as

$$
v_{l}=\mu_{l+1}-\mu_{l} ; v_{N}=\mu_{1}-\mu_{N}+1 .
$$

Traditionally the first $N-1$ dyons are called the 'M - dyons' and the $N^{-t h}$ dyon is called the ' $\mathrm{L}$ - dyon', because an additional gauge transformation is need for it to have correct asymptotics.

We also introduce a notation

$$
\mathrm{v}_{m n} \equiv 2 \pi\left(\mu_{m}-\mu_{n}\right) \bmod 2 \pi .
$$

which coincides with $\mathrm{v}=\mathrm{v}_{21}$ and $\overline{\mathrm{v}}=\mathrm{v}_{12}$ used previously in the $S U(2)$ calculations.

\footnotetext{
${ }^{3}$ We use notations consistent with [5].
} 
The positions of dyon centers are denoted by $y_{i}$. The distance from the $i^{- \text {th }}$ dyon center to a point $x$ is denoted as $\vec{x}-\vec{y}_{i}=r_{i}$; for the $S U(2)$ case the standard notation is $r_{1}=s ; r_{2}=r$ [15]. The distance between dyon cores is denoted by $r_{i j}=\left|\vec{r}_{j}-\vec{r}_{i}\right|$.

It is convenient to use a so-called 'algebraic gauge', in which the asymptotic gauge field is vanishing at the expense of introducing twisted boundary conditions for field fluctuations. The twist $a(\vec{x}, 1 / T)=e^{-i \tau} a(\vec{x}, 0)$ is hence related to the holonomy as $\tau=2 \pi \operatorname{diag}\left(\mu_{1}, \ldots, \mu_{N}\right)$. The holonomy and, correspondingly, the twist could also be multiplied by elements of the center of the gauge group $e^{2 \pi i \frac{k}{N}}$. It does not affect the adjoint gauge field and determinant but it affects fundamental determinants [17]:

$$
\begin{aligned}
\log \operatorname{det}\left(-\nabla_{N}^{2}\right)= & \sum_{n}\left(\frac{\pi}{4} P^{\prime \prime}\left(\tau_{n}\right) r_{n, n-1}+\frac{1}{2} P\left(\tau_{n}\right) V^{(3)}-\frac{v_{n} \log v_{n}}{6}-\frac{\log r_{n, n-1}}{12 \pi r_{n, n-1}}\right) \\
& +c_{N}+\frac{1}{6} \log \mu+\mathcal{O}(1 / r)
\end{aligned}
$$

where

$$
c_{N}=-\frac{13}{72}-\frac{\pi^{2}}{216}+\frac{\log \pi}{6}-\frac{\zeta^{\prime}(2)}{\pi^{2}} .
$$

$P$ is a periodical function with a period $2 \pi$ such that

$$
P(\mathrm{v})=\frac{q^{2}(2 \pi-q)^{2}}{12 \pi^{2}} ; q=\bmod 2 \pi
$$

Determinant in the adjoint representation of $S U(2)$ reads $[15,19]$

$$
\begin{aligned}
& \log \operatorname{Det}\left(-D_{2}^{2}\right)=V P(\mathrm{v})+2 \pi P^{\prime \prime}(\mathrm{v}) r_{12}+\frac{3 \pi-4 \mathrm{v}}{3 \pi} \log \mathrm{v}+\frac{3 \pi-4 \overline{\mathrm{v}}}{3 \pi} \log \overline{\mathrm{v}} \\
& +\frac{2}{3} \log \mu+\frac{5}{3} \log (2 \pi)+c_{2} \\
& +\frac{1}{r_{12}}\left[\frac{1}{\mathrm{v}}+\frac{1}{\overline{\mathrm{v}}}+\frac{23 \pi}{54}-\frac{8 \gamma_{E}}{3 \pi}-\frac{74}{9 \pi}-\frac{4}{3 \pi} \log \left(\frac{\mathrm{v} \overline{\mathrm{v}} r_{12}^{2}}{\pi^{2}}\right)\right]+\mathcal{O}\left(\frac{1}{r_{12}^{2}}\right)
\end{aligned}
$$

Now we proceed to the calculation of $S U(N)$ adjoint-representation determinant.

\section{Method of computation}

For self-dual fields the gluonic and ghost determinants over non-zero modes for the background gauge fixing are related [27] to the adjoint scalar determinant in the same background: $\operatorname{Det}^{\prime}\left(W_{\mu \nu}\right)=\operatorname{Det}\left(-D^{2}\right)^{4}$, where $W_{\mu \nu}$ is the quadratic form for spin-1, adjoint representation quantum fluctuations and $D^{2}$ is the covariant Laplace operator for spin-0, adjoint representation ghost fields. So the total contribution to the effective action of gluon and ghost determinants is $2 \log \operatorname{Det}\left(-D^{2}\right)$ which corresponds to two physical degrees of freedom.

We calculate the quantum determinant by integrating its variation with respect to parameters $\mathcal{P}$ of the solution, following $[17,16,15,28]$. In this case the problem reduces to four dimentional integral of the gauge field variation multiplied by a vacuum current, 
which can be expressed through Green function known implicitly for any self-dual configuration

$$
\frac{\partial \log \operatorname{Det}\left(-D^{2}[A]\right)}{\partial \mathcal{P}}=-\int d^{4} x \operatorname{Tr}\left(\partial_{\mathcal{P}} A_{\mu} J_{\mu}\right)
$$

where $J_{\mu}$ is the vacuum current in the external background, determined by the Green function:

$$
J_{\mu} \equiv \vec{D}_{\mu} \mathcal{G}+\mathcal{G} \overleftarrow{D}_{\mu}
$$

Here $\mathcal{G}$ is the periodical Green function of the covariant Laplas operator in adjoint representation

$$
\begin{aligned}
-D_{x}^{2} G(x, y) & =\delta^{(4)}(x-y) \\
\mathcal{G}(x, y) & =\sum_{n=-\infty}^{+\infty} G\left(x_{4}, \vec{x} ; y_{4}+n, \vec{y}\right) .
\end{aligned}
$$

The Green functions in the self-dual backgrounds are known explicitly $[29,30]$ if the gauge field is expressed in terms of the Atiyah-Drinfeld-Hitchin-Manin (ADHM) construction [31]: $A_{\mu}=v^{\dagger} \partial_{\mu} v$. These look quite simple for the fundamental representation [32]

$$
G^{\text {fund }}(x, y)=\frac{v^{\dagger}(x) v(y)}{4 \pi^{2}(x-y)^{2}},
$$

but become more complicated for the adjoint representation [33, 34, 35]:

$$
\begin{aligned}
G^{a b}(x, y) & =\frac{\frac{1}{2} \operatorname{Tr} t^{a}\langle v(x) \mid v(y)\rangle t^{b}\langle v(y) \mid v(x)\rangle}{4 \pi^{2}(x-y)^{2}} \\
& +\frac{1}{4 \pi^{2}} \int_{-1 / 2}^{1 / 2} d z_{1} d z_{2} d z_{3} d z_{4} M\left(z_{1}, z_{2}, z_{3}, z_{4}\right) \\
& \times \frac{1}{2} \operatorname{Tr}\left(\mathcal{V}^{\dagger}\left(x, z_{1}\right) \mathcal{V}\left(x, z_{2}\right) t^{a}\right) \operatorname{Tr}\left(\mathcal{V}^{\dagger}\left(y, z_{4}\right) \mathcal{V}\left(y, z_{3}\right) t^{b}\right)
\end{aligned}
$$

where $t^{a}$ are Hermitian fundamental-representation generators of $S U(N)$ normalized to $\operatorname{tr} t^{a} t^{b}=\frac{1}{2} \delta^{a b} ; \mathcal{V}(x, z)$ is one of the components of $v$ (see eq.(65)) and $M$ is a piecewise rational function ${ }^{4}$. Fortunately we do not need an explicit form of this function for the $S U(N)$ caloron since in the large separation limit the contribution of the last term (or "M-term") is exponentially small away from the dyons. Near the dyons the field is essentially reduced to the $S U(2)$, so one can use there the results of [34, 15]. In what follows it will be convenient to split the periodic propagator into three parts and consider them separately:

$$
\begin{aligned}
\mathcal{G}(x, y)= & \mathcal{G}^{\mathrm{r}}(x, y)+\mathcal{G}^{\mathrm{s}}(x, y)+\mathcal{G}^{\mathrm{m}}(x, y) \\
& \mathcal{G}^{\mathrm{s}}(x, y) \equiv G(x, y) \\
& \mathcal{G}^{\mathrm{r}}(x, y)+\mathcal{G}^{\mathrm{m}}(x, y) \equiv \sum_{n \neq 0} G\left(x_{4}, \vec{x} ; y_{4}+n, \vec{y}\right)
\end{aligned}
$$

\footnotetext{
${ }^{4}$ see Appendix B to [15] for its explicit form in case of $S U(2)$
} 
here $\mathcal{G}^{\mathrm{m}}(x, y)$ coresponds to the part of the propagator arising from the $M$-term. The vacuum current (9) will be also split into three parts, "singular" , "regular" and "M", in accordance with (14)

$$
J_{\mu}=J_{\mu}^{\mathrm{r}}+J_{\mu}^{\mathrm{s}}+J_{\mu}^{\mathrm{m}} .
$$

As was proposed in [15] we divide the space into regions surrounding the dyons and the remaining space (outer region). Near each of the dyons the gauge field becomes essentially the $S U(2)$ dyon configuration plus an additional constant-field background. In this region we can use the results of [15]. In the outer region, far from the exponential cores of the dyons, the vacuum current considerably simplifies and we only have to perform integration in (8).

In the following two sections we give results for these two domains and in section 5.1 we combine them together and integrate over the space.

\section{Core domain}

In this section we write a contribution to the variation of total determinant arising from the core region of a dyon. We take a ball of radius $R$ around the dyon. In that region the field is approximately the one of the $S U(2)$-dyon, embedded along one of the simple roots, plus an extra constant $A_{4}$ field [5]. More precisely in the fundamental representation the gauge field near the $l^{\text {th }}$ dyon is a zero $N \times N$ matrix with only $2 \times 2$ block at $l^{- \text {th }}$ position filled by the BPS dyon gauge field, plus a constant diagonal $N \times N$ matrix [17]

$$
\begin{aligned}
& A_{\mu}^{l^{\text {th }} \text { block } 2 \times 2}=A_{\mu}^{\text {dyon }}\left(v_{l}, \vec{x}-\vec{y}_{l}\right)+2 \pi i\left(\frac{\mu_{l}+\mu_{l+1}}{2}\right) \delta_{\mu 4} 1_{2 \times 2}, \\
& A_{\mu}^{\text {outside } l^{\text {th }} \text { block } 2 \times 2}=2 \pi i \operatorname{diag}\left(\mu_{1}, \ldots, \mu_{N}\right) \delta_{\mu 4} .
\end{aligned}
$$

Under the action of the $S U(2)$ sub-group, the adjoint representation of $S U(N)$ splits into one triplet, $2(N-2)$ doublets and $(N-2)^{2}$ singlets. The determinant of arbitrary $S U(2)$ configuration embedded into $S U(N)$ is then expressed as a sum of $S U(2)$ adjoint-representation determinant plus $2(N-2)$ fundamental-representation determinants ${ }^{5}$.

As is seen from eq.(16), the $S U(2)$ dyon field is accomplished by the constant diagonal matrix. This matrix can be killed by a gauge transformation, which is not periodical, and thus can change the determinant. It is equivalent to the additional twist of boundary conditions for the $2(N-2)$ fundamental representation determinants.

As a demonstration let us consider the $S U(3)$ case. The fundamental gauge field reads

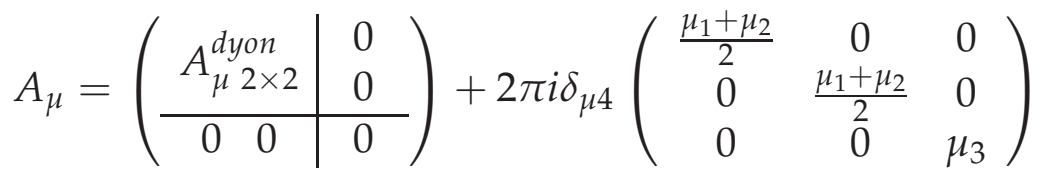

\footnotetext{
${ }^{5}$ See for example [36] where that was done for the instanton solution.
} 
in the adjoint representation of $S U(3)$ in an appropriate basis it becomes

$$
\hat{A}_{\mu}=\left(\begin{array}{c|c|c|c}
\hat{A}_{\mu 3 \times 3}^{\text {dyon }} & 0 & 0 & 0 \\
\hline 0 & -A_{\mu 2 \times 2}^{\text {dyon }}-i \pi \delta_{\mu 4}\left(v_{3}-v_{2}\right) & 0 & 0 \\
\hline 0 & 0 & A_{\mu 2 \times 2}^{\text {dyon }}+i \pi \delta_{\mu 4}\left(v_{3}-v_{2}\right) & 0 \\
\hline 0 & 0 & 0 & 0
\end{array}\right)
$$

So that there is one block $3 \times 3$ giving adjoint representation dyon field and two $2 \times 2$ blocks, giving fundamental representation dyon accomplished by a unit matrix. As it was shown in [17] this extra unit matrix changes only an IR divergent part of the dyon determinant (the one, depending on radius of the ball). These divergences cancel with the terms in the outer-region determinant depending on the radius of the holes $R$, as it is shown in Appendix A). So we can freely drop them.

Summing up one adjoint (42) and 2(N-2) fundamental dyon determinants (41) for all $N$ dyons we obtain the following contribution to the derivative of the Caloron determinant from the considered domain

$$
\partial_{\mathcal{P}} \sum_{n}\left(-\frac{(6+N) v_{n} \log \left(v_{n}\right)}{3}+\log \left(v_{n}\right)\right)+\mathrm{IR},
$$

where "IR" denotes the IR divergent terms.

\section{Outer domain}

We proceed to consider the far domain, i.e. the region of space outside dyons' cores. Caloron field becomes diagonal with $\mathcal{O}\left(e^{-v_{i} r_{i}}\right)$ precision and this simplifies significantly the results. For instance the $4^{\text {th }}$ component of the fundamental caloron gauge field reads

$$
A_{4}^{m n}=i \delta^{m n}\left(2 \pi \mu_{m}+\frac{1}{2 r_{m}}-\frac{1}{2 r_{m-1}}\right)
$$

In what follows we will consider the derivative of the determinant with respect to $\mu_{m}$. It turns out that in this domain only $A_{4}$ depends on $\mu_{m}$ nontrivially [5]. Thus we need only $4^{\text {th }}$ component of the vacuum current as it follows from (8). As we know from the $S U(2)$ case this component of the current is especially simple [15]

$$
J_{4}^{\mathrm{su}(2)}=\frac{i}{2} T_{3} P^{\prime}\left(v+\frac{1}{r_{1}}-\frac{1}{r_{2}}\right)
$$

the natural generalization of this expression is

$$
J_{4}^{\mathrm{su}(\mathrm{n})}=\underset{n, m=1}{N}\left[\frac{i}{2} P^{\prime}\left(2 \pi\left(\mu_{m}-\mu_{n}\right)+\frac{1}{2 r_{m}}-\frac{1}{2 r_{m-1}}-\frac{1}{2 r_{n}}+\frac{1}{2 r_{n-1}}\right)\right]
$$

The expression in the brackets is simply the eigenvalue of the the gauge field (20) in the adjoint representation. This formula is definitely right for large $r_{m}$, where the field 
becomes almost constant $[2,37]$ and generalizes the $S U(2)$ expression. Moreover we check it by a direct computation in Appendix B. We conclude that

$$
-\operatorname{tr}\left(\partial_{\mathcal{P}} A_{\mu} J_{\mu}\right)=\frac{1}{2} \sum_{n, m} \partial_{\mathcal{P}} P\left(\mathrm{v}_{m n}+\frac{1}{2 r_{m}}-\frac{1}{2 r_{m-1}}-\frac{1}{2 r_{n}}+\frac{1}{2 r_{n-1}}\right)
$$

where

$$
\mathrm{v}_{m n} \equiv 2 \pi\left(\mu_{m}-\mu_{n}\right)
$$

The variation over $\mathcal{P}$ can be integrated up to a constant, and the integral over space would be performed in the next section.

\subsection{Integration}

In order to get a variation of the determinant we have to integrate in eq.(23) over the space with $N$ spherical holes of radius $\mathrm{R}$. The following integrals will be very helpful

$$
\begin{aligned}
& \int\left(\frac{1}{2 r_{m}}-\frac{1}{2 r_{m-1}}-\frac{1}{2 r_{n}}+\frac{1}{2 r_{n-1}}\right)^{2} d^{3} x \simeq \\
& \quad \pi\left(r_{m, n}+r_{m-1, n-1}-r_{m, n-1}-r_{m-1, n}+r_{m, m-1}+r_{n, n-1}\right) \\
& \int\left(\frac{1}{2 r_{m}}-\frac{1}{2 r_{m-1}}-\frac{1}{2 r_{n}}+\frac{1}{2 r_{n-1}}\right)^{3} d^{3} x \simeq \\
& \quad-3 \pi \log \left(\frac{r_{m, n-1}}{r_{n, m-1}} \frac{r_{m, n, m-1}}{r_{n, m, n-1}} \frac{r_{n, m-1, n-1}}{r_{m, n-1, m-1}}\right)
\end{aligned}
$$

where $r_{n m}=\left|\vec{y}_{n}-\vec{y}_{m}\right|$ is a distance between dyons and

$$
2 r_{l m n} \equiv r_{l m}+r_{m n}+r_{n l}
$$

is the perimeter of the triangle, formed by $l^{- \text {th }}, m^{- \text {th }}, n^{- \text {th }}$ dyons. Sign $\simeq$ means that we drop all the terms dependent on the radius of the holes $R$ since they cancels precisely with dyons IR divergences as discussed in Appendix A. To derive the last equation we used

$$
\int \frac{1}{r_{n} r_{m} r_{l}} d^{3} x \simeq-4 \pi \log r_{n m l}+C .
$$

It is important to point out that the eq.(26) is not applicable for the case $m=n \pm 1$, since it diverges. The reason is that the divergences near dyon cores are not balanced anymore. Nevertheless it is straightforward to verify that if one replaces a zero $r_{n n}$ under logarithm in eq.(26) by some fixed $\epsilon$, then it is is still valid up to a constant, which cancels in the final result.

So we can integrate in eq.(23)

$$
\begin{aligned}
& \int \frac{1}{2} \sum_{m, n} P\left(\mathrm{v}_{m n}+\frac{1}{2 r_{m}}-\frac{1}{2 r_{m-1}}-\frac{1}{2 r_{n}}+\frac{1}{2 r_{n-1}}\right) d^{3} x \simeq \\
& \quad \sum_{m, n} \frac{\pi}{4} P^{\prime \prime}\left(\mathrm{v}_{m n}\right)\left(r_{m, n}+r_{m-1, n-1}-r_{m, n-1}-r_{m-1, n}+r_{m, m-1}+r_{n, n-1}\right) \\
& \quad-\sum_{m, n} \frac{\left[\mathrm{v}_{m n}\right]-\pi}{2 \pi} \log \left(\frac{r_{m, n-1}}{r_{n, m-1}} \frac{r_{m, n, m-1}}{r_{n, m, n-1}} \frac{r_{n, m-1, n-1}}{r_{m, n-1, m-1}}\right)+\sum_{n, m} \frac{1}{2} P\left(\mathrm{v}_{m n}\right) V^{(3)}
\end{aligned}
$$


We denote $\left[\mathrm{v}_{m n}\right]=\mathrm{v}_{m n}$ mod $2 \pi$. To simplify the above expression we use the identity:

$$
\sum_{m, n} \frac{\left[\mathbf{v}_{m n}\right]-\pi}{4 \pi} \log \frac{r_{m, n, m-1} r_{n, m-1, n-1}}{r_{n, m, n-1} r_{m, n-1, m-1}}=\sum_{m, n} v_{n} \log r_{m, n, m-1}-\sum_{n} \log r_{n, n-1} .
$$

Then eq.(28) becomes

$$
\begin{aligned}
& \log \operatorname{det}\left(-D_{N}^{2}\right)^{f a r}=\int \frac{1}{2} \sum_{m, n} P\left(\mathrm{v}_{m n}+\frac{1}{2 r_{m}}-\frac{1}{2 r_{m-1}}-\frac{1}{2 r_{n}}+\frac{1}{2 r_{n-1}}\right) d^{3} x \simeq \\
& \quad-\sum_{m, n} \frac{\left[\mathrm{v}_{m n}\right]-\pi}{2 \pi} \log \left(\frac{r_{m, n-1}}{r_{n, m-1}}\right)-\sum_{m, n} 2 v_{n} \log r_{m, n, m-1}+2 \sum_{n} \log r_{n, n-1}+ \\
& \quad \sum_{m, n} \frac{\pi}{4} P^{\prime \prime}\left(\mathrm{v}_{m n}\right)\left(r_{m, n}+r_{m-1, n-1}-r_{m, n-1}-r_{m-1, n}+r_{m, m-1}+r_{n, n-1}\right)+ \\
& \quad+\sum_{m, n} \frac{1}{2} P\left(\mathrm{v}_{m n}\right) V^{(3)}
\end{aligned}
$$

The "R-terms" are exactly the ones of eq.(44) but with $R$ standing as a lower limit of integration, this provides the cancellation of them when we add the core contribution. The second equality in (30) is valid when the variation does not involve changing of the far region. Note that the $\frac{\log r}{r}$ correction comes only from the far region. So we can calculate it. It comes from the next $P^{\prime \prime \prime \prime}$ term in the Taylor series, this term obviously involves 4-center Coulomb integrals:

$$
\int \frac{d^{3} x}{r_{1} r_{2} r_{3} r_{4}}
$$

taken over $\mathrm{R}^{3}$ with holes around centers. Since this integral converges both in the IR and UV (near the holes), it can involve only logarithms of some dimensionless combinations of distances between these four points, divided by the distance. In the approximation that the dyons are spread homogeneously, such terms would be of order of unity and we neglect them. The only large logarithms come from the case where three of the four points coincide, in this case the integral diverges as logarithm near the $i$-th dyon:

$$
\int_{\mathrm{R}^{4} \backslash B_{R}} \frac{1}{r_{i}^{3} r_{j}}=4 \pi \frac{\log \left(r_{i j} / R\right)}{r_{i j}}+\mathcal{O}\left(1 / r_{i j}\right)
$$

So for the correction to $\log \operatorname{Det}\left(-D^{2}\right)$ one sums all the contributions of the form (32). Note that $P^{\mathrm{IV}}=\frac{2}{\pi}$ is a constant, so some terms cancel in the sum. The result for $N>2$ is:

$$
\log \operatorname{Det}\left(-D^{2}\right)_{\text {correction }}=-\frac{6+N}{6 \pi} \sum_{n>m}^{N} \frac{\log r_{n m}}{r_{n m}},
$$

for $N=2$ the coefficient is doubled and becomes $-\frac{8}{3 \pi}$, since there are more coincident points. It matches our $S U(2)$ result (eq. (60) in [15] ). 


\section{The result}

From eqs. $(19,30)$ we can conclude that for large dyons' separations, $\varrho_{m} \ll 1 / \nu_{m}+$ $1 / v_{m-1}$, the $\operatorname{SU}(N)$ caloron determinant is the sum of these expressions plus some integration constant and $\frac{\log r}{r}$ improvement (33). Restoring the temperature dependence we obtain

$$
\begin{aligned}
& \log \operatorname{det}\left(-D_{N}^{2}\right)= \\
& -\sum_{m, n} \frac{\left[\mathrm{v}_{m n}\right]-\pi}{2 \pi} \log \left(\frac{r_{m, n-1}}{r_{n, m-1}}\right)-\sum_{m, n} 2 v_{n} \log r_{m, n, m-1}+2 \sum_{n} \log r_{n, n-1} \\
& +\sum_{m, n} \frac{\pi}{4} P^{\prime \prime}\left(\mathrm{v}_{m n}\right) T\left(r_{m, n}+r_{m-1, n-1}-r_{m, n-1}-r_{m-1, n}+r_{m, m-1}+r_{n, n-1}\right) \\
& +\sum_{m, n} \frac{1}{2} P\left(\mathrm{v}_{m n}\right) T^{3} V^{(3)}-\sum_{n} \frac{(6+N) v_{n} \log v_{n}}{3}+\sum_{n} \log v_{n}-\frac{6+N}{6 \pi} \frac{\log r_{n m} T}{r_{n m} T}+c_{N}
\end{aligned}
$$

Note that the coefficient $-\frac{6+N}{6 \pi}$ should be doubled for $N=2$ case.

The contribution to the effective action from non-zero modes of gluons and ghosts would be

$$
\delta S_{e f f}=-\log \frac{\operatorname{Det}\left(-D^{2}\right)}{\left(\operatorname{Det}^{\prime} W_{\mu \nu}\right)^{1 / 2}}=\log \operatorname{Det}\left(-D^{2}\right)
$$

The constant $c_{N}$ will of course contain a standard UV-divergence $c_{N}=c+\frac{N}{3} \log \mu_{P V}$, coming from the instanton determiant [38], where $\mu_{P V}$ is a Pauli-Villars mass. This divergence, together with $\left(\frac{\mu_{P V}}{g \sqrt{2 \pi}}\right)^{4 N}$ coming from zero modes, gives the standard YangMills $\beta$-function and is commonly incorporated into the running coupling:

$$
\mu_{P V}^{\frac{11}{3} N} e^{-\frac{8 \pi^{2}}{8^{2}\left(\mu_{P V}\right)}}=\Lambda^{\frac{11}{3} N}
$$

where $\Lambda$ is the scale parameter obtained here through the 'transmutation of dimensions'.

Now let us combine with the result for $\operatorname{SU}(N)$ caloron zero modes [23, 22] and the classical action $8 \pi^{2} / g^{2}\left(\mu_{P V}\right)$. The caloron measure is [23]

$$
\int_{\mathcal{G}} \omega \simeq 2^{6 N} \pi^{4 N}\left[1+\sum_{m} \frac{1}{4 \pi \varrho_{m}}\left(\frac{1}{v_{m-1}}+\frac{1}{v_{m}}\right)\right] \prod_{n} v_{n} d^{3} \varrho_{1} \ldots d^{3} \varrho_{N-1} d^{4} \xi .
$$

So the total contribution of one caloron to the effective action becomes

$$
\begin{aligned}
e^{-S_{e f f} \approx} & \left(\frac{\Lambda e^{\gamma_{E}}}{4 \pi T}\right)^{\frac{11}{3} N} C_{N} \int\left(\operatorname{Det}\left(-D_{N}^{2}\right)\right)^{-1}\left(\frac{8 \pi^{2}}{g^{2}\left(\mu_{P V}\right)}\right)^{2 N} \times \\
& \times\left[1+\sum_{m} \frac{1}{4 \pi \varrho_{m}}\left(\frac{1}{v_{m-1}}+\frac{1}{v_{m}}\right)\right] \prod_{n} v_{n} d^{3} \varrho_{1} \ldots d^{3} \varrho_{N-1} d^{4} \xi
\end{aligned}
$$

We have collected the factor $4 \pi e^{-\gamma_{E}} T / \Lambda$ because it is the natural argument of the running coupling constant at nonzero temperatures $[39,37]$. When we have done so in the $S U(2)$ case [15], we have got a constant numerically very close to 1 , so we expect the constant $C$ to be of order of unity. 


\section{Acknowledgements}

I thank Nikolay Gromov for collaboration, discussions and editing this work. We are grateful to Dmitri Diakonov, Victor Petrov, Konstantin Zarembo and Michael MullerPreussker for discussions. This work was partially supported by RSGSS-1124.2003.2 and by RFFI project grant 06-02-16786, the Dmitri Zimin 'Dynasty' foundation, STINT Institutional Grant and by a Grant from VR.

\section{A Cancellation of IR divergenses of dyons}

Consider the field near the dyon constituent of $S U(N)$ caloron. In the fundamental representation it is given by the eq.(16). In the adjoint representation this field looks like one block with the $S U(2)$ BPS dyon in the $S U(2)$-adjoint representation $(3 \times 3)$ and $2(N-2)$ blocks with the SU(2) BPS dyon in the SU(2)-fundamental representation $(2 \times 2)$ plus a constant part, specified below:

$$
\begin{aligned}
-i A_{\text {const }}^{\text {adj }}= & \pi \operatorname{diag}\left(0,0,0,-\left(\mu_{1}+\mu_{2}-2 \mu_{3}\right),-\left(\mu_{1}+\mu_{2}-2 \mu_{3}\right),\right. \\
& +(. .),+(. .), \ldots,+\left(\mu_{1}+\mu_{2}-2 \mu_{N}\right),+\left(\mu_{1}+\mu_{2}-2 \mu_{N}\right), \\
& \left., \mu_{3}-\mu_{4}, \mu_{4}-\mu_{3}, . .\left(\text { all pairs without } \mu_{1}, \mu_{2}\right) \ldots, 0,0, . .\right)
\end{aligned}
$$

There are $N-2$ zeroes in the end, corresponding to elements of the Cartan subalgebra other than $T^{3}$. Let us check the size of the matrix (39):

$$
3+4(N-2)+(N-2)(N-3)+(N-2)=N^{2}-1
$$

as it should be for the adjoint representation of $S U(N)$.

This constant background is exactly equivalent to twisting the boundary conditions. So we have to sum logarithms of determinants for one adjoint-representation $S U(2)$ dyon, 2(N-2) differently twisted fundamental-representation $S U(2)$ dyons and a determinant for $(N-2)(N-3)$ different constant $A_{4}$ field eigenvalues. It would be interesting to check that this is asymptotically the same as the adjoint determinant for the far region that we will calculate below.

In [17] we proved that the twisting of boundary conditions for the fundamental representation determinant results in shifting of the argument of $P$, where $P$ is the standard perturbative potential

$$
P(\mathrm{v})=\left.\frac{1}{3(2 \pi)^{2}} \mathrm{v}^{2}(2 \pi-\mathrm{v})^{2}\right|_{\bmod 2 \pi} .
$$

Now we can just write the result (for the first dyon, for simplicity), as a sum of the formula taken from [15] for the triplet (SU(2) adjoint representation):

$$
\begin{aligned}
& \partial_{\mathcal{P}} \log \operatorname{Det}\left(-D^{2}\right)_{\text {near dyon }} \\
& =\partial_{\mathcal{P}}\left(\tilde{c}_{\text {dyon }} \nu_{1}-\frac{8}{3} v_{1} \log \left(\nu_{1}\right)+\log v_{m}+\int^{R} P\left(2 \pi v_{1}-\frac{1}{r}\right) 4 \pi r^{2} d r\right)
\end{aligned}
$$


with 2(N-2) formulas from [17] for the $S U(2)$ dyon with twisted boundary conditions ("twist" is a corresponding matrix element of eq.(39)) ${ }^{6}$ :

$$
\begin{aligned}
& \partial_{\mathcal{P}} \log \operatorname{Det}\left(-\nabla^{2}\right)_{\text {near dyon }}=\sum_{i=1}^{2(N-2)} \partial_{\mathcal{P}}\left\{\hat{c}_{\text {dyon }} \nu_{1}-\frac{\log \left(v_{1}\right)}{6} v_{1}\right. \\
& +\int^{R} \frac{1}{2}\left[P\left(2 \pi\left(\mu_{1}-\left(\mu_{1}+\mu_{2}\right) / 2\right)+\frac{1}{2 r}-i\left(A_{\text {const }}^{\text {adj }}\right)_{2 i+1}\right)\right. \\
& \left.\left.+P\left(2 \pi\left(\mu_{2}-\left(\mu_{1}+\mu_{2}\right) / 2\right)-\frac{1}{2 r}-i\left(A_{\text {const }}^{\text {adj }}\right)_{2 i+2}\right)\right] 4 \pi r^{2} d r\right\}
\end{aligned}
$$

and with a constant-field determinant for the remaining matrix elements of $-D^{2}$ (i.e. twists without background field)

$$
\partial_{\mathcal{P}} \log \operatorname{Det}\left(-D^{2}\right)_{\text {near dyon }}^{\text {const }}=\sum_{i=1}^{(N-2)(N-3)} \partial_{\mathcal{P}} \int_{0}^{R} \frac{1}{2} P\left(-i\left(A_{\text {const }}^{\text {adj }}\right)_{2+4(N-2)+i}\right) 4 \pi r^{2} d r .
$$

Totally we get:

$$
\begin{aligned}
\partial_{\mathcal{P}} \log \operatorname{Det}( & \left.-D^{2}\right)_{\text {near dyon }}=\partial_{\mathcal{P}}\left(c_{\text {dyon }} v_{m}-\left(\frac{8}{3}+\frac{N-2}{3}\right) \log \left(v_{m}\right) v_{m}+\log v_{m}\right) \\
+(R-\text { terms }) &
\end{aligned}
$$

And it is easy to check explicitly that the "R-terms" exactly match the asymptotics of far-from-dyons domain (see the Section 5)

$$
(R \text {-terms })=\lim _{r_{i \neq 1} \rightarrow \infty} \int^{R} \sum_{i>j} \delta P\left[2 \pi\left(\mu_{i}-\mu_{j}\right)+\frac{1}{2 r_{i}}-\frac{1}{2 r_{i-1}}-\left(\frac{1}{2 r_{j}}-\frac{1}{2 r_{j-1}}\right)\right] 4 \pi r_{1}^{2} d r_{1}
$$

So we conclude that the " $\mathrm{R}$ - terms" are trivial and exactly match the contributions from the outer region, as it should be, of course, since the result cannot depend on the radius of the auxiliary balls that we have chosen.

\section{B Calculation of the currents for outer domain}

\section{B.1 Singular current}

The contribution of the singular part of the propagator to the variation of the determinant is 4 times the fundamental representation result $[27,2]$, if we write this variation in terms of fundamental representation. So we just take our old result from [17] (that formula was not written there explicitly, it was in our intermediate computations). For the component $J^{\mathrm{si}}$ it is quite natural to introduce bipolar spatial coordinates with unit

\footnotetext{
${ }^{6}$ Note that to get the normal $S U(2)$ dyon we have to center the interval $\left(\mu_{1}, \mu_{2}\right)$ at zero, because in $S U(2)$ we obviously have the zero-trace condition $\mu_{1}+\mu_{2}=0$
} 
repers $\hat{r}_{i}=\frac{r_{i}}{|r|}, \hat{s}_{i}=\frac{r_{i-1}}{|r|}, n_{\phi}=\frac{\hat{r} \times \hat{s}}{|\hat{r} \times \hat{s}|}$. In these coordinates the current (already multiplied by 4 ) is

$$
\begin{aligned}
J_{4}^{\mathrm{si}} & =-\frac{i\left(r_{i}^{3}-s_{i}^{3}\right)}{12 \pi^{2} r_{i}^{3} s_{i}^{3}} \\
J_{\phi}^{\mathrm{si}} & =-\frac{i\left(r_{i}+s_{i}\right) \sqrt{-\left(\varrho_{i}-r_{i}-s_{i}\right)\left(d+r_{i}-s_{i}\right)\left(\varrho_{i}-r_{i}+s_{i}\right)\left(\varrho_{i}+r_{i}+s_{i}\right)}}{4 \pi^{2} r_{i}^{2} s_{i}^{2}\left(\varrho_{i}+r_{i}+s_{i}\right)^{2}} \\
J_{\hat{r}_{i}}^{\mathrm{si}} & =0 ; \quad J_{\hat{s}_{i}}^{\mathrm{si}}=0
\end{aligned}
$$

We also remind the notations: $r_{i}=x-y_{i}$ is a vector from the $i$-th dyon center to the current point, $s_{i} \equiv r_{i-1}$; and $\varrho_{i}=\left|y_{i}-y_{i-1}\right|$ is a distance between these two dyons. Also standard "circular rule" $r_{N+1} \equiv r_{1}$ is implied.

\section{B.2 M-term current}

Let us prove that the contribution to the current from the M-term of adjoint propagator is zero with exponential precision (i.e. it decays exponentially out of the dyon cores). As was shown in [15], when making the propagator periodic the M-term simplifies to

$$
\mathcal{G}^{\mathrm{m} a b}(x, y) \equiv \frac{1}{8 \pi^{2}} \int_{-1 / 2}^{1 / 2} d z d z^{\prime} M\left(z, z^{\prime}\right) \operatorname{Tr}\left(v^{2 \dagger}(x, z) v^{2}(x, z) \tau^{a}\right) \operatorname{Tr}\left(v^{2 \dagger}\left(y, z^{\prime}\right) v^{2}\left(y, z^{\prime}\right) \tau^{b}\right)
$$

since the property $v\left(y^{n}, z\right)=e^{2 \pi i n z} v(y, z)$ used to derive that result, still holds for the $\operatorname{SU}(N)$ ADHMN construction. Here $M\left(z_{1}, z_{2}, z, z\right)=\delta\left(z_{1}-z_{2}\right) M\left(z_{1}, z\right)$.

First of all we note that only the lower components of $v$ are left and only the Cartan (diagonal) components are nonzero: From eq.(78) we see that for each $m$ the function $s_{m}(z)$ and hence $v_{m}^{2}(z)$ is peaked near $z=\mu_{m}$ and exponentially decays away from this point. So $v_{m}^{2+}(x, z) v_{n}^{2}(x, z) \sim \delta_{m n}$ with exponential precision. This leads us to conclusion that

$$
\mathcal{G}^{\mathrm{m} a b}(x, y) \propto \delta^{a \in \operatorname{Cartan}} \delta^{b \in \text { Cartan }}, \quad \mathcal{G}^{\mathrm{m} a b}(x, y)=\mathcal{G}^{\mathrm{m} a b}(y, x) .
$$

The second equation means that the terms with derivatives in the expression for the current (9) cancel each other. It follows from the first one that the adjoint action of $A$ on $\mathcal{G}^{m}$ gives zero since both lie approximately in the Cartan subalgebra. Therefore we conclude that

$$
J_{\mu}^{\mathrm{m}} \simeq 0
$$

\section{B.3 Regular current}

The adjoint-representation regular current is

$$
J^{a b}=D_{x}^{a c} \mathcal{G}_{c b}(x, y)+\mathcal{G}_{a c}(x, y) D_{y}^{c b}
$$


where $a, b, c=1 . . N^{2}-1$ and we take the regular part of the propagator:

$$
\left(\mathcal{G}^{\mathrm{r}}\right)^{a b}(x, y) \equiv \sum_{n \neq 0} \frac{4}{8 \pi^{2}\left(x-y_{n}\right)^{2}} \operatorname{Tr}\left[t^{a}\left\langle v(x) \mid v\left(y_{n}\right)\right\rangle t^{b}\left\langle v\left(y_{n}\right) \mid v(x)\right\rangle\right], \quad y_{n}^{i}=y^{i}-\delta^{i 4} n,
$$

It is possible to rewrite these formulae in the fundamental notations and evaluate explicitly. Some details of the calculation together with a short review of ADHM construction are presented below. We denote the adjoint indices by $a, b, c=1 . . N^{2}-1$ and fundamental indices by $i, j, k, l, m, n=1 . . N$.

First we represent the covariant derivatives in the fundamental representation. With the help of the identities

$$
\begin{aligned}
& \vec{D}_{\mu}^{a d} \operatorname{tr}\left(t^{d} A t^{b} B\right)=\operatorname{tr}\left[t^{a}\left(\vec{D}_{\mu} A\right) t^{b} B-t^{a} A t^{b}\left(B \overleftarrow{D}_{\mu}\right)\right] \\
& \operatorname{tr}\left(t^{a} A t^{d} B\right) \overleftarrow{D}_{\mu}^{d b}=\operatorname{tr}\left[t^{a}\left(A \overleftarrow{D}_{\mu}\right) t^{b} B-t^{a} A t^{b}\left(\vec{D}_{\mu} B\right)\right]
\end{aligned}
$$

one gets for $J^{a b}$ the obvious four terms plus $\delta_{\mu 4} \frac{2 \operatorname{tr}\left[t^{a} v_{x}^{\dagger} v_{y} t^{b} v_{y}^{\dagger} v_{x}\right]}{\pi^{2} n^{3}}$ from the derivative acting on the denominator.

All the terms in the adjoint current have the form $\operatorname{tr}\left[t^{a} B t^{b} C\right]$. The variation of the determinant has the form $-\delta A^{c} T_{a b}^{c} J^{a b}$. To write it in the fundamental representation we use the identities:

$$
A^{c} T_{a b}^{c}=2 \operatorname{tr}\left(t^{b}\left[t^{a}, A\right]\right)
$$

where $A=A^{i} t^{i}$ and

$$
t_{i j}^{a} t_{k l}^{a}=1 / 2\left(\delta_{i l} \delta_{j k}-1 / N \delta_{i j} \delta_{k l}\right)
$$

The Hermitian generators $t^{a}$ are normalized as $\operatorname{tr}\left(t^{a} t^{b}\right)=1 / 2$ (for $\operatorname{SU}(2)$ these are $\left.\tau^{a} / 2\right)$. We get:

$$
\delta A^{c} T_{a b}^{c} \operatorname{tr}\left(t^{a} B t^{b} C\right)=\frac{1}{2} \operatorname{tr}(B) \operatorname{tr}(\delta A C)-\frac{1}{2} \operatorname{tr}(C) \operatorname{tr}(\delta A B)
$$

So in terms of the fundamental indices $(i, j=1 \ldots N)$ we get for the current $J^{i j}$ (that is to be coupled to $A$ in the fundamental representation to get the variation of determinant)

$$
\begin{aligned}
\left(J_{\mu}^{\mathrm{r}}\right)^{i j}(x)= & \sum_{n \neq 0,\{B, C\}} \frac{1}{4 \pi^{2}\left(x-y_{n}\right)^{2}}\left(\operatorname{tr}(B) C^{i j}-\operatorname{tr}(C) B^{i j}\right) \\
& -\delta_{\mu 4} \sum_{n \neq 0} \frac{1}{\pi^{2}\left(x-y_{n}\right)^{3}}\left(\operatorname{tr}(E) F^{i j}-\operatorname{tr}(F) E^{i j}\right)
\end{aligned}
$$

Here

$$
E=v_{x}^{\dagger} v_{y_{n}} \equiv b ; F=v_{y_{n}}^{\dagger} v_{x} \equiv b^{\dagger}
$$

and we put $y=x$ (so that now $y_{n}^{i}=x^{i}-\delta^{i 4} n$ ) according to eq.(9). The set $\{(B, C)\}$ consists of 4 pairs taken from eq.(53) and eq.(51):

$$
\{(B, C)\}=\left\{\left(D_{x} v_{x}^{\dagger} v_{y_{n}}, v_{y_{n}}^{\dagger} v_{x}\right),\left(v_{x}^{\dagger} v_{y_{n}}, D_{x} v_{y_{n}}^{\dagger} v_{x}\right),\left(v_{x}^{\dagger} v_{y_{n}} D_{y}, v_{y_{n}}^{\dagger} v_{x}\right),\left(v_{x}^{\dagger} v_{y_{n}}, v_{y_{n}}^{\dagger} v_{x} D_{y}\right)\right\}
$$


Since the current and the field are approximately diagonal in the fundamental representation, we consider the diagonal components $\left(J_{\mu}^{\mathrm{r}}\right)^{i} \equiv\left(J_{\mu}^{\mathrm{r}}\right)^{i i}$. In these notations the contribution to equation eq.(57) can be rewritten as

$$
\left(J^{\mathrm{r}}{ }_{\mu}\right)^{i}(x)=\sum_{j=1}^{N} \sum_{n \neq 0}\left(\sum_{\{(B, C)\}} \frac{1}{4 \pi^{2}\left(x-y_{n}\right)^{2}}\left(B_{j} C_{i}-C_{j} B_{i}\right)-\frac{\delta_{\mu 4}}{\pi^{2}\left(x-y_{n}\right)^{3}}\left(E_{j} F_{i}-F_{j} E_{i}\right)\right)
$$

From eq.(59) and eq.(60) we get $8+2$ terms in the resulting contribution to the current. Now in order to calculate explicitly these $B, C, E, F$ we need ADHMN construction. A brief review and a calculation follows.

\section{B.4 Expressions of the ADHM construction}

The basic object in the ADHMN construction $[31,30]$ is the $(2+N) \times 2$ matrix $\Delta$ linear in the space-time variable $x$ and depending on an additional compact variable $z$ belonging to the unit circle:

$$
\Delta_{\beta}^{K}(z, x)=\left\{\begin{array}{cl}
\lambda_{\beta}^{m}(z) & , \quad K=m, \quad 1 \leq m \leq N, \\
\left(B(z)-x_{\mu} \sigma_{\mu}\right)_{\beta}^{\alpha}, \quad K=N+\alpha, \quad 1 \leq \alpha \leq 2,
\end{array}\right.
$$

where $\alpha, \beta=1,2$ and $m=1, \ldots, N ; \sigma_{\mu}=\left(i \vec{\sigma}, 1_{2}\right)$. As usual, the superscripts number rows of a matrix and the subscripts number columns. The functions $\lambda_{\beta}^{m}(z)$ forming a $N \times 2$ matrix carry information about color orientations of the constituent dyons, encoded in the $N$ two-spinors $\zeta$ :

$$
\lambda_{\beta}^{m}(z)=\delta\left(z-\mu_{m}\right) \zeta_{\beta}^{m} .
$$

The quantities $\zeta_{\beta}^{m}$ transform as contravariant spinors of the gauge group $S U(N)$ but as covariant spinors of the spatial $S U(2)$ group. The $2 \times 2$ matrix $B$ is a differential operator in $z$ and depends on the positions of the dyons in the $3 d$ space $\vec{y}_{m}$ and the overall position in time $\xi_{4}=x_{4}$ :

$$
B_{\beta}^{\alpha}(z)=\frac{\delta_{\beta}^{\alpha} \partial_{z}}{2 \pi i}+\frac{\hat{A}_{\beta}^{\alpha}(z)}{2 \pi i}
$$

with

$$
\hat{A}(z)=A_{\mu} \sigma_{\mu}, \quad \vec{A}(z)=2 \pi i \vec{y}_{m}(z), \quad A_{4}=2 \pi i \xi_{4},
$$

where inside the interval $\mu_{m} \leq z \leq \mu_{m+1}, \vec{y}(z)=\vec{y}_{m}$ is the position of the $m^{\text {th }}$ dyon with inverse size $v_{m} \equiv \mu_{m+1}-\mu_{m}$.

One has to find $N$ quantities $v_{n}^{K}(x), n=1 \ldots N$,

$$
v_{n}^{K}(x)=\left\{\begin{array}{cl}
v_{n}^{1 m}(x) & , \quad K=m, \quad 1 \leq m \leq N \\
v_{n}^{2 \alpha}(z, x) & , \quad K=N+\alpha, \quad 1 \leq \alpha \leq 2,
\end{array}\right.
$$

which are normalized independent solutions of the differential equation

$$
\lambda^{+\alpha}(z) v_{n}^{1 m}+\left[B^{\dagger}(z)-x_{\mu} \sigma_{\mu}^{\dagger}\right]_{\beta}^{\alpha} v_{n}^{2 \beta}(z, x)=0, \quad v_{l}^{+1 m} v_{n}^{1 l}+\int_{-1 / 2}^{1 / 2} d z v_{\alpha}^{+2 m} v_{n}^{2 \alpha}=\delta_{n}^{m},
$$


or, in short hand notations,

$$
\Delta^{\dagger} v=0, \quad v^{\dagger} v=1_{N}
$$

Note that only the lower component $v^{2}$ depends on $z$.

Expressing $v$ as

$$
v(x)=\left(\begin{array}{c}
-1_{n} \\
u(x)
\end{array}\right) \phi^{-1 / 2}, \quad u(x)=\left(B^{\dagger}-x^{\dagger}\right)^{-1} \lambda^{\dagger}
$$

let us find $u(z, x)$ - the main object of ADHM construction. It is the solution to the equation

$$
\left(B^{\dagger}-x^{\dagger}\right) u=\lambda^{\dagger}, \quad B^{\dagger}-x^{\dagger}=\frac{\partial_{z}}{2 \pi i}-r^{\dagger}(z)
$$

Define the Green's functions:

$$
f=\left(\Delta^{\dagger} \Delta\right)^{-1}, \quad G=\left((B-x)^{\dagger}(B-x)\right)^{-1}, \quad \phi_{i j}(x)=\delta_{i j}+\lambda_{i}^{\alpha} G^{\alpha \beta} \lambda_{j}^{+\alpha}
$$

where $(i=1 \ldots N),(\alpha, \beta=1,2)$. One can note that

$$
f=\left(G^{-1}+\lambda^{\dagger} \lambda\right)^{-1}=G-G \lambda_{i}^{\dagger} \phi_{i j}^{-1} \lambda G
$$

acting on (71) with $\lambda^{\dagger}$ on the right yields

$$
G \lambda_{j}^{\dagger}=f \lambda_{i}^{\dagger} \phi_{i j}
$$

The Green's function is expressed as follows:

$$
\begin{gathered}
f\left(z, z^{\prime}\right)=s_{m}(z) f_{m n} s_{n}^{\dagger}\left(z^{\prime}\right)+2 \pi s\left(z, z^{\prime}\right) \delta_{[z]\left[z^{\prime}\right]} \\
f_{m n}=F^{-1}{ }_{m n}
\end{gathered}
$$

The functions appearing in eq.(73) are

$$
\begin{gathered}
s_{m}(z)=e^{2 \pi i x_{0}\left(z-\mu_{m}\right)} \frac{\sinh \left[2 \pi r_{m}\left(\mu_{m+1}-z\right)\right]}{\sinh \left(2 \pi r_{m} v_{m}\right)} \delta_{m[z]}+e^{2 \pi i x_{0}\left(z-\mu_{m}\right)} \frac{\sinh \left[2 \pi r_{m-1}\left(z-\mu_{m-1}\right)\right]}{\sinh \left(2 \pi r_{m-1} v_{m-1}\right)} \delta_{m,[z]+1}, \\
s\left(z, z^{\prime}\right)=e^{2 \pi i x_{0}\left(z-z^{\prime}\right)} \frac{\sinh \left(2 \pi r_{[z]}\left(\min \left\{z, z^{\prime}\right\}-\mu_{[z]}\right)\right) \sinh \left(2 \pi r_{[z]}\left(\mu_{[z]+1}-\max \left\{z, z^{\prime}\right\}\right)\right)}{r_{[z]} \sinh \left(2 \pi r_{[z]} v_{[z]}\right)} \\
u_{i}=(B-x) f \lambda_{j}^{\dagger} \phi_{j i}=\left(\frac{\partial_{z}}{2 \pi i}-r_{\mu} \sigma_{\mu}\right) s_{k}^{f}(z) f_{k j} \zeta_{j}^{\dagger} \phi_{j i}
\end{gathered}
$$

And the convenient notation is $r_{i}=x-y_{i}$ is a vector from the $i$-th dyon to the current point. First we note that $F_{i j}$ and $\phi_{i j}$ are diagonal matrices with exponential precision

$$
f_{i j} \simeq 2 \pi \delta_{i j}\left(r_{i}+r_{i-1}+\varrho_{i}\right)^{-1}
$$




$$
\phi_{i j} \simeq \delta_{i j} \frac{r_{i}+r_{i-1}+\varrho_{i}}{r_{i}+r_{i-1}-\varrho_{i}}
$$

To pass to the periodical gauge we multiply $v(x)$ from the right by $g_{i j}=\delta_{i j} e^{2 \pi i \mu_{i} x_{0}}$. Totally within the exponential precision we get for $v$ :

$$
v_{i}(x, z)=\left(\begin{array}{c}
-\delta_{i j} \phi_{i i}^{-1 / 2} e^{2 \pi i \mu_{i} x_{0}} \\
(B-x) s_{i}(z) f_{i i} \zeta_{i}^{\dagger} \phi_{i i}^{1 / 2} e^{2 \pi i \mu_{i} x_{0}}
\end{array}\right) \text { no index summations }
$$

Consider the covariant derivative of $v_{i}(x)$ in the periodical gauge (integration over $z$ is assumed):

$$
\begin{aligned}
D_{\mu}\langle v(x)| & =\partial_{\mu}\langle v|-\partial_{\mu}\langle v \mid v\rangle\langle v|=\partial_{\mu}\langle v|(1-|v\rangle\langle v|) \\
& =\partial_{\mu}\langle v| \Delta f \Delta^{\dagger}=-\langle v| \partial_{\mu} \Delta f \Delta^{\dagger} \\
& =-\langle v| \mathcal{B} \sigma_{\mu} f \Delta^{\dagger}=\left(v^{2 \dagger} \sigma_{\mu} f \lambda^{\dagger}, v^{2 \dagger} \sigma_{\mu} f(B-x)^{\dagger}\right) \\
& =f_{i i} \zeta_{i} \phi_{i i}^{1 / 2} e^{-2 \pi i \mu_{i} x_{0}}(B-x)^{\dagger} s_{i}^{\dagger}(z) \sigma_{\mu}\left(f \lambda^{\dagger}, f(B-x)^{\dagger}\right) \\
& =f_{i i} \zeta_{i} \phi_{i i}^{1 / 2} e^{-2 \pi i \mu_{i} x_{0}}(B-x)^{\dagger} s_{i}^{\dagger}(z) \sigma_{\mu}\left(s_{i}(z) f_{i i} \zeta_{i}^{\dagger},\left(s_{i}(z) f_{i i} s_{i}^{\dagger}\left(z^{\prime}\right)+2 \pi s\left(z, z^{\prime}\right)\right)(B-x)^{\dagger}\right) . \\
& |v(x)\rangle D_{\mu}=-\left(D_{\mu}\langle v(x))^{\dagger}\right.
\end{aligned}
$$

\section{B.5 Formula for the regular current}

Let us denote $D=(B-x)$. We will in a moment express eq.(59) through $c$ and $b$, defined as

$$
\begin{aligned}
c & =D_{\mu} v_{x}^{\dagger} v_{y} \\
& =-\phi n f_{i i}^{2} \zeta_{i}(D s(z))^{\dagger} \sigma_{\mu}\left(s(z) f s^{\dagger}\left(z^{\prime}\right)+2 \pi s\left(z, z^{\prime}\right)\right) e^{-2 \pi i n\left(z^{\prime}-\mu_{i}\right)} D s\left(z^{\prime}\right) \zeta_{i}^{\dagger} e^{-2 \pi i \mu_{i} n} \\
& =-\phi n f_{i i}^{2} \zeta_{i}(\tilde{D} \tilde{s}(z))^{\dagger} \sigma_{\mu}\left(\tilde{s}(z) f \tilde{s}^{\dagger}\left(z^{\prime}\right)+2 \pi \tilde{s}\left(z, z^{\prime}\right)\right) \tilde{D} \tilde{s}\left(z^{\prime}\right) \zeta_{i}^{\dagger} e^{-2 \pi i n z^{\prime}}
\end{aligned}
$$

here $\sim$ means that the time dependence is separated (so that $\tilde{s}$ is time-independent) and integration over $z$ and $z^{\prime}$ is assumed. To derive this we used

$$
D_{x}^{+} v_{y}^{2}=\left(D_{y}^{+}-n\right) v_{y}^{2}=-\lambda v_{y}^{1}-n v_{y}^{2}
$$

following from eq.(67) and noticed that the first term cancels with the scalar product of upper components. For $b$ we easily get

$$
b=v_{x}^{\dagger} v_{y}=\exp \left(-2 \pi i \mu_{i} n\right) / \phi_{i}+\phi_{i} f_{i i}^{2} \zeta_{i}\left((D s)^{\dagger} e^{-2 \pi i n z} D s\right) \zeta_{i}^{+} .
$$

We also need the following formulas:

$$
\begin{aligned}
v_{x}^{\dagger} v_{y} D_{\mu}^{(y)} & =-c_{n \rightarrow-n}^{\dagger} \\
v_{y}^{\dagger} v_{x} & =b^{\dagger} \\
b_{n \rightarrow-n} & =b^{\dagger} \\
v_{y}^{\dagger} v_{x} D_{\mu}^{(y)} & =-D_{\mu}^{(y)} v_{y}^{\dagger} v_{x}+2 A_{\mu} v_{y}^{\dagger} v_{x}=-c_{n \rightarrow-n}+2 A b^{\dagger} \\
D_{\mu}^{(x)} v_{y}^{\dagger} v_{x} & =-v_{y}^{\dagger} v_{x} D_{\mu}^{(x)}+2 A v_{y}^{\dagger} v_{x}=c^{\dagger}+2 A b^{\dagger}
\end{aligned}
$$


In the total sum changing $n \rightarrow-n$ does not affect the expression since we divide by $n^{2}$, so we can make $n \rightarrow-n$ in the whole expression by conjugating $b$ and then drop $n \rightarrow-n$.

Then the set of $\{(B, C)\}$ in eq.(59) becomes

$$
\{(B, C)\}=\left\{\left(c, b^{\dagger}\right),\left(b, c^{\dagger}+2 A b^{\dagger}\right),\left(-c_{n \rightarrow-n}^{\dagger}, b^{\dagger}\right),\left(b,-c_{n \rightarrow-n}+2 A b^{\dagger}\right)\right\}
$$

Totally we get for the current (60) (the index $\mu$ is hidden in $c$ ):

$$
\left(J^{\mathrm{r}}{ }_{\mu}\right)^{i}(x)=\sum_{n \neq 0 ; j=1 . . N} \frac{2 c_{j} b_{i}^{\dagger}-2 c_{j}^{\dagger} b_{i}-4 A_{j} b_{i} b_{j}^{\dagger}}{4 \pi n^{2}}-\delta_{\mu 4} \frac{b_{j} b_{i}^{\dagger}}{\pi^{2} n^{3}}-(i \leftrightarrow j)
$$

There are only terms with $(i) \neq(j)$ in the current that we are calculating.

Now by these explicit formulas the current can be evaluated by performing integrals over $z, z^{\prime}$ and summing over $n$. The reference formulas for summation can be found e.g. in [15]. We used Mathematica for these calculations. Below the result is presented. Just for illustration and consistency check we write first for the $S U(2)$. For $S U(2)$ the ADHMN data is taken to be $\mu_{1}=-\omega, \mu_{2}=\omega, v_{1}=2 \omega, v_{2}=1-2 \omega$. So we get for the current (here we write the diagonal matrix, which couples to the gauge field in the fundamental representation)

$$
\left(J_{4}^{r}\right)^{1}=-\left(J_{4}^{r}\right)^{2}=\left(i P^{\prime}\left(2 \pi\left(\mu_{1}-\mu_{2}\right)+\frac{1}{r}-\frac{1}{s}\right)+\frac{i}{12 \pi^{2}}\left(\frac{1}{s^{3}}-\frac{1}{r^{3}}\right)\right)
$$

As usual, the current is expressed as a derivative of perturbative potential $P$. The second term in this expression cancels exactly with the contribution of "singular" current. Totally, adding the singular current, we get for the "far region" contribution to variation:

$$
\begin{aligned}
\delta \log \operatorname{Det}\left(-D^{2}\right)_{f a r} & =\int_{f a r} \delta\left(A_{41}^{\text {fund }}-A_{42}^{\text {fund }}\right) P^{\prime}\left(2 \pi\left(\mu_{1}-\mu_{2}\right)+\frac{1}{r}-\frac{1}{s}\right) \\
& =\int_{f a r} \delta P\left(2 \pi\left(\mu_{1}-\mu_{2}\right)+\frac{1}{r}-\frac{1}{s}\right)
\end{aligned}
$$

we remind the convenient notation: $r_{i}=x-y_{i}$ is a vector from the $i$-th dyon to the current point, $s_{i} \equiv r_{i-1}$ and a standard "circular rule" $r_{N+1} \equiv r_{1}$. For $S U(2)$ we set $r=r_{1}, s=s_{1}=r_{2}$.

For the $S U(N)$ case we get the total result:

$$
\begin{aligned}
& \delta \log \operatorname{Det}\left(-D^{2}\right)_{f a r} \\
= & \int_{f a r} \sum_{i, j=1}^{N} \delta\left(2 \pi \mu_{i}+\frac{1}{2 r_{i}}-\frac{1}{2 s_{i}}\right) P^{\prime}\left[2 \pi\left(\mu_{i}-\mu_{j}\right)+\frac{1}{2 r_{i}}-\frac{1}{2 s_{i}}-\left(\frac{1}{2 r_{j}}-\frac{1}{2 s_{j}}\right)\right] \operatorname{sgn}\left(\mu_{i}-\mu_{j}\right) \\
= & \int_{f a r} \sum_{i>j} \delta P\left[2 \pi\left(\mu_{i}-\mu_{j}\right)+\frac{1}{2 r_{i}}-\frac{1}{2 s_{i}}-\left(\frac{1}{2 r_{j}}-\frac{1}{2 s_{j}}\right)\right]
\end{aligned}
$$

Note that the spatial part of the regular current cancelled exactly with the singular part of the current. This proves the eq.(23). 


\section{References}

[1] A. M. Polyakov, Phys. Lett. B 72, 477 (1978).

[2] D.J. Gross, R.D. Pisarski and L.G. Yaffe, Rev. Mod. Phys. 53, 43 (1981).

[3] A. Belavin, A. Polyakov, A. Schwartz and Yu. Tyupkin, Phys. Lett. 59, 85 (1975).

[4] T.C. Kraan and P. van Baal, Phys. Lett. B 428, 268 (1998) 268, hep-th/9802049; Nucl. Phys. B 533, 627 (1998), hep-th/9805168.

[5] T. C. Kraan and P. van Baal, Phys. Lett. B 435, 389 (1998) [arXiv:hep-th/9806034].

[6] K. Lee and C. Lu, Phys. Rev. D 58, 025011 (1998), hep-th/9802108.

[7] F. Bruckmann and P. van Baal, Nucl. Phys. B 645, 105 (2002) [arXiv:hepth/0209010].

[8] F. Bruckmann, D. Nogradi and P. van Baal, Nucl. Phys. B 698, 233 (2004) [arXiv:hep-th/0404210].

[9] E.M. Ilgenfritz, B.V. Martemyanov, M. Muller-Preussker, S. Shcheredin and A.I. Veselov, Phys. Rev. D 66, 074503 (2002), hep-lat/0206004;

E. M. Ilgenfritz, B. V. Martemyanov, M. Muller-Preussker, S. Shcheredin and A. I. Veselov, Nucl. Phys. Proc. Suppl. 119, 754 (2003) [arXiv:hep-lat/0209081];

E. M. Ilgenfritz, B. V. Martemyanov, M. Muller-Preussker and A. I. Veselov, Phys. Rev. D 69, 114505 (2004) [arXiv:hep-lat/0402010];

[10] P. van Baal, arXiv:hep-ph/0610409.

[11] D. Diakonov and V. Petrov , arXiv:0704.3181

[12] B.J. Harrington and H.K. Shepard, Phys. Rev. D 17, 2122 (1978); ibid. 18, 2990 (1978).

[13] K. Lee and P. Yi, Phys. Rev. D 56, 3711 (1997), [hep-th/9702107].

[14] K. Lee, E.J. Weinberg and P. Yi, Phys. Lett. B376 (1996) 97 [hep-th/9601097]; Phys. Rev. D54 (1996) 6351 [hep-th/9605229]; E.J. Weinberg, Massive and Massless Monopoles and Duality, hep-th/9908095.

[15] D.Diakonov, N.Gromov, V.Petrov and S.Slizovskiy, Phys. Rev. D 70036003 (2004)

[16] N. Gromov and S. Slizovskiy, Phys. Rev. D 71, 125019 (2005) [arXiv:hepth/0504024].

[17] N. Gromov and S. Slizovskiy, Phys. Rev. D 73, 025022 (2006) [arXiv:hepth/0507101].

[18] N. Gromov, Prepared for 58th Scottish Universities Summer School in Physics (SUSSP58): A NATO Advanced Study Institute and EU Hadron Physics 13 Summer Institute, St. Andrews, Scotland, 22-29 Aug 2004 
[19] N. Gromov, arXiv:hep-th/0701192.

[20] R. C. Brower, D. Chen, J. Negele, K. Orginos and C. I. Tan, Nucl. Phys. Proc. Suppl. 73, 557 (1999), hep-lat/9810009.

[21] C. Gattringer, Phys. Rev. D 67, 034507 (2003) [arXiv:hep-lat/0210001]; C. Gattringer et al., Nucl. Phys. Proc. Suppl. 129 (2004) 653 [arXiv:hep-lat/0309106]; C. Gattringer and R. Pullirsch, Phys. Rev. D 69, 094510 (2004) [arXiv:heplat/0402008].

[22] T. C. Kraan, Commun. Math. Phys. 212, 503 (2000) [arXiv:hep-th/9811179].

[23] D. Diakonov and N. Gromov, Phys. Rev. D 72, 025003 (2005) [arXiv:hepth/0502132].

[24] M. N. Chernodub, T. C. Kraan and P. van Baal, Nucl. Phys. Proc. Suppl. 83, 556 (2000) [arXiv:hep-lat/9907001].

[25] N. Weiss, Phys. Rev. D 24, 475 (1981); ibid. D25, 2667 (1982).

[26] P. Gerhold, E. M. Ilgenfritz and M. Muller-Preussker, Nucl. Phys. B 760, 1 (2007) [arXiv:hep-ph/0607315]; arXiv:hep-ph/0610426.

[27] L.S. Brown and D.B. Creamer, Phys. Rev. D 18, 3695 (1978).

[28] K. Zarembo, Nucl. Phys. B 463, 73 (1996), hep-th/9510031.

[29] N.H. Christ, E.J. Weinberg and N.K. Stanton, Phys. Rev. D 18, 2013 (1978);

E. Corrigan, P. Goddard and S. Templeton, Nucl. Phys. B 151, 93 (1979).

[30] W. Nahm, Phys. Lett. B 90, 413 (1980).

[31] M.F. Atiyah, V.G. Drinfeld, N.J. Hitchin and Yu.I. Manin, Phys. Lett. A 65, 185 (1978).

[32] E. Corrigan, D. B. Fairlie, S. Templeton and P. Goddard, Nucl. Phys. B 140, 31 (1978).

[33] E. Corrigan, D. B. Fairlie, S. Templeton and P. Goddard, Nucl. Phys. B 140, 31 (1978).

[34] W. Nahm, Phys. Lett. B 90, 413 (1980).

[35] I. Jack, Nucl. Phys. B 174, 526 (1980).

[36] C. W. Bernard, N. H. Christ, A. H. Guth and E. J. Weinberg, Phys. Rev. D 16, 2967 (1977).

[37] D. Diakonov and M. Oswald, Phys. Rev. D 68, 025012 (2003) [arXiv:hepph/0303129]; Phys. Rev. D 70, 016006 (2004) [arXiv:hep-ph/0312126]; Phys. Rev. D 70, 105016 (2004) [arXiv:hep-ph/0403108].

[38] G. 't Hooft, Phys. Rev. D 14, 3432 (1976). 
[39] S. Huang and M. Lissia, Nucl. Phys. B438 (1995) 54;

K. Kajantie, M. Laine, K. Rummukainen and M.E. Shaposhnikov, Nucl. Phys. B503 (1997) 357;

S. Chapman, Phys. Rev. D50 (1994) 5308. 